\section{Recursos para la redacción científica de artículos de investigación en salud}

\author{
Resources for the scientific \\ writing of health research articles
}

Manuel Antonio Mattos-Vela ${ }^{1, a, b}$, Luis Cuadrao Zavaleta ${ }^{1, a, c}$

${ }^{1}$ Facultad de Odontología. Universidad Nacional Mayor de San Marcos. Lima, Perú.

a Doctor en Estomatología

${ }^{\text {b }}$ Editor general de la revista Odontología Sanmarquina

c Director de la revista Odontología Sanmarquina

Correspondencia:

Manuel Antonio Mattos-Vela

Correo electrónico: mmattosv@unmsm.edu.pe

Facultad de Odontología, UNMSM. Calle Germán Amézaga 375.

Lima 1, Perú.

Coautor:

Luis Cuadrao Zavaleta

lcuadraoz@unmsm.edu.pe

Fecha de recepción: 26/01/18

Fecha de aceptación: 30/01/18
La ciencia crece por medio de los conocimientos generados por la investigación científica. Esta investigación tiene que ser transmitida a la comunidad científica pero también a la población en general y el canal formal para realizarlo es a través de las revistas científicas indizadas.

Existe evidencia que mucho de los artículos publicados en estas revistas son deficientes en calidad y claridad de la comunicación ${ }^{1}$. Además, muchos manuscritos enviados para su posible publicación a una revista son rechazados por lo incompleto y poca claridad de su contenido. Muchas veces falta información relevante para poder valorar la calidad de la investigación. Por ello, es que hace más de 20 años, en el campo de la salud empezaron a publicarse directrices para informar correctamente los trabajos de investigación (reporting guidelines). Estas directrices o pautas, son consensos realizados por expertos en metodología de la investigación, estadística, informes y trabajo editorial, que orientan cómo debemos redactar y qué información debemos comunicar de acuerdo a los diferentes diseños de investigación. Seguir estas directrices permite elaborar artículos de alta calidad, facilita la revisión por pares y aumenta la posibilidad que un manuscrito sea aceptado por una revista científica ${ }^{2}$.

Es fundamental que todos los investigadores y profesionales de la salud que desean publicar artículos científicos conozcan estas directrices. Todas ellas las podemos encontrar reunidas en la Red EQUATOR ${ }^{3}$ (enancing the quality and transparency of health research) la cual alberga actualmente cerca de 400 directrices, además cuenta con una serie de recursos para producir publicaciones de investigación de alta calidad y está dirigida a los autores de artículos de investigación, editores de revistas científicas, revisores pares y a quienes realizan directrices de publicación. Los recursos son de acceso libre y regularmente son actualizados para asegurar la disponibilidad de las últimas pautas para informar.

Si bien la Red EQUATOR fue lanzada oficialmente el 2008 en el Reino Unido y ha tenido una labor fructífera, aun no es completamente conocida por todos los involucrados en las publicaciones científicas ni es lo suficientemente consultada, a pesar de la riqueza de recursos para la publicación que ofrece a la comunidad científica del área de la salud ${ }^{4}$. Por ello, este editorial busca informar sobre las herramientas que están actualmente disponibles para mejorar los artículos de investigación de tal manera que sean más completos, precisos y transparentes. Existen directrices generales para informes con diferentes tipos de estudio ${ }^{3,5}$, tales como: CONSORT (para informes de ensayos clínicos aleatorios), STROBE (para estudios observacionales), PRISMA (para revisiones sistemáticas y metanálisis), STARD (para estudios de precisión diagnóstica), CARE (para reportes de caso clínico), COREQ y ENTREQ (para investigación cualitativa) y ARRIVE (para estudios preclínicos en anima- 
les) ${ }^{3}$. Estas directrices generales deberían ser siempre consideradas cuando se reportan este tipo de estudios.

Sin embargo, la mayoría de las directrices que podemos encontrar en el sitio web de EQUATOR ${ }^{3}$, son más específicas, muchas de ellas son extensiones de las directrices generales, por ejemplo, entre las extensiones de CONSORT existe una aplicada y adaptada para ensayos clínicos de ortodoncia, también se presenta una directriz para informar resúmenes de ensayos aleatorios en revistas y congresos. La directriz CARE tiene como extensión a SCARE (para reportar casos quirúrgicos), entre otros más. Asimismo, otras de las directrices específicas son consensos sobre algunos tipos de estudios en diferentes disciplinas o en diferentes secciones del informe ${ }^{3}$. Por ejemplo, en el campo de la Odontología encontramos estándares para informar estudios epidemiológicos sobre prevalencia y severidad de la periodontitis crónica. Estas directrices específicas deberían ser usadas idealmente en combinación con las directrices enfocadas en la metodología genérica.

Odontología Sanmarquina recomienda en sus directrices para autores que los manuscritos que sean enviados para revisión consulten la Red EQUATOR y de manera especial las directrices para informes que ella contiene.

Asimismo, este año nuestra revista desea contribuir con sus lectores dando una orientación para que mejoren la redacción de sus artículos, por ello en cada fascículo del 2018 estaremos publicando, en la sección de Notas
Científicas, un artículo que aborda el tema de redacción científica. Estas recomendaciones están basadas en diversos textos y las directrices antes mencionadas. En este número se presentan pautas para la redacción del Título, Resumen y Palabras clave; en el fascículo de junio se publicará una nota relacionada a la Introducción y Métodos, en setiembre sobre Resultados y en diciembre sobre la Discusión.

\section{Referencias bibliográficas}

1. Simera I. Get the content right: Following reporting guidelines will make your research paper more complete, transparent and usable. J Pak Med Assoc. 2013;63(2):283-5.

2. Simera I, Altman DG. Reporting medical research. Int J Clin Pract. 2013;67:701-2. doi:10.1111/ijcp.12168

3. EQUATOR Network. Library for Health Research Reporting. [Consultado el 25 de enero 2018]. Accesible en: http://www.equator-network.org/.

4. Reveiz L, Villanueva E, Iko Ch, Simera I. Compliance with clinical trial registration and reporting guidelines by Latin American and Caribbean journals. Cad Saúde Pública. 2013;29(6):1095-1100.

5. International Committee of Medical Journal Editors. Recommendations for the conduct, reporting, editing, and publication of scholarly work in medical journals (updated december 2017). [Consultado el 25 de enero 2018]. Accesible en: http://www.icmje.org/recommendations/. 